\title{
PRODUCT INTEGRAL SOLUTIONS FOR HEREDITARY SYSTEMS
}

\author{
BY
}

JAMES A. RENEKE

\begin{abstract}
Hereditary systems which satisfy a Lipschitz condition are solved in terms of product integrals. Realizations of this type of hereditary system are provided from functional differential and integral equations.
\end{abstract}

Introduction. Suppose that $\{X,\|\cdot\|\}$ is a Banach space, $S$ is an interval of nonnegative numbers which includes $0, S^{\prime}$ is an interval of numbers which contains $S$, and $G$ is a linear space of functions from $S^{\prime}$ into $X$. We will use 0 to denote, where appropriate, either the real number, the zero element of $X$, or the member of $G$ whose only value is the zero of $X$. Suppose that $N$ is a function from $S$ into the class of all pseudonorms on $G$ such that

(i) If $w$ is in $S$ and $f$ is in $G$ then $N_{w}(f)=0$ if and only if $f(u)=0$, for all $u \leq w$,

(ii) if $u \leq w$ then $N_{u}(f) \leq N_{w}(f)$, for each $f$ in $G$, and

(iii) $\{G, N\}$ is complete.

Let $H$ denote the class of all functions from $G$ into $G$ and 1 the identity function in $H$. Suppose that $K$ is a function from $S^{\prime} \times S^{\prime}$ into $H$ such that

(i) if $f$ is in $G,[u, v]$ is a subinterval of $S^{\prime}$, and $t$ is a member of $S^{\prime}$ not exceeding $u$, then $[K(u, v) f](t)=0$,

(ii) if each of $u, v$, and $w$ is in $S^{\prime}$ and $v$ is either between $u$ and $w, u$ $=v$, or $v=w$, then $K(u, v) f+K(v, w) f=K(u, w) f$, for each $f$ in $G$, and

(iii) there is a function $k$ from $S$ into the class of nondecreasing functions from $S$ to the numbers such that, for each positive number $w$ in $S$,

$$
N_{w}(K(u, v) 0) \leq k_{w}(v)-k_{w}(u)
$$

and

$$
N_{w}(K(u, v) f-K(u, v) g) \leq(L) \int_{u}^{v} N_{x}(f-g) d k_{w}(x)
$$

for each $(f, g)$ in $G \times G$ and subinterval $[u, v]$ of $[0, w]$.

We want to consider the equation

Presented to the Society, November 19, 1971; received by the editors March 14, 1972 and, in revised form, November 9, 1972.

AMS (MOS) subject classifications (1970). Primary 45D05; Secondary 45N05, 93 A10. Key words and phrases. Hereditary system, product integral, functional equation. 


$$
b(u)=f(u)+[K(0, u) b](u),
$$

where $f$ is in G. Equation (1) will be called an bereditary system. The phrase hereditary system is meant to suggest a system for which the response depends only upon the previous history of the system. For Stieltjes integral equations [7] the phrase well posed has been used to describe this situation. Amongst functional differential and integral equations [3] those which exhibit this behavior are called delay equations. Nonanticipatory is used for stochastic integral equations [9]. Hale and Cruz [4] applied the phrase hereditary system to a class of such systems described using derivatives. There is a large overlap of equation (1) with the systems discussed in each of the se areas. Our purpose is to apply the method of product integration, which may be loosely described as a refinement of the Cauchy-Euler method, to the problems of existence, uniqueness, and continuity for hereditary systems.

Existence and uniqueness. We should note that if $b$ is in $G$ then the function $g$ from $S^{\prime}$ into $X$ defined by $g(u)=[K(0, u) b](u)$, for all $u$ in $S^{\prime}$, is in $G$. This follows from the completeness of $G$. Let $\hat{g}$ denote the function from $S$ into $G$ defined by $\hat{g}_{u}=K(0, u) b$, for each $u$ in $S$. Then $N_{w}\left(\hat{g}_{u}-\hat{g}_{v}\right)=0$, for $u, v$, and $w$ in $S$ and $w \leq u \leq v$. Hence the net $\{S, \hat{g}\}$ has a limit $g_{1}$ in $G$. But $g_{1}(u)=\hat{g}_{w}(u)=[K(0, w) b](u)=g(u)$, for $w$ in $S$ and $u$ in $S^{\prime}$ not exceeding $w$. So $g$ is in $G$ and $g(u)=0$, for $u$ in $S^{\prime}$ not exceeding 0 .

Thus for each $f$ in $G$ we seek a solution of equation (1) in $G$. Our method for exhibiting such a solution is essentially that of [11] and depends upon the introduction of a related operator equation. We will introduce and use Mac Nerney's device [8] of jumping the dimension in order to circumvent the technical difficulty that members of $H$ might not map 0 to 0 .

Let $\biguplus=G \times I(2)$, where $I(2)$ denotes the integers modulo 2 . Define addition on $\biguplus$ componentwise and $\gamma_{\text {on }} \varrho$ by $\gamma_{u}((g, m))=N_{u}(g)+m$, for $u$ in $S$ and $(g, m)$ in $\mathcal{S}_{\text {. }}$ Let $\mathcal{H}$ denote the functions from $\oint$ into $\varrho$ which map the zero of $\oint$ to zero. We will also use 1 to denote the identity function in $\mathcal{H}$. Let $V$ denote the function from $S \times S$ into $H$ defined as follows:

$$
\left[V(u, v)_{g}\right](w)= \begin{cases}{[K(v, u) g](w)} & \text { if } v \leq u, \\ 0 & \text { otherwise, }\end{cases}
$$

for each $g$ in $G,(u, v)$ in $S \times S$, and $w$ in $S^{\prime}$. Let $Z^{0}$ denote the function from $S \times S$ into $\mathcal{H}$ defined by $\mathcal{C}(u, v)(g, m)=(V(u, v) g-V(u, v) 0+m V(u, v) 0,0)$, for each $(u, v)$ in $S \times S$ and $(g, m)$ in $\zeta$.

Suppose that $k$ is a function from $S$ into the class of nondecreasing functions from $S$ to the numbers corresponding to $K$ as in condition (iii). Such a function $k$ will be called a family of super functions for $K$. 
Theorem 1. For each $(u, v)$ in $S \times S$ and $g$ in $G$, the continuously continued product $\Pi^{v}[1+V] g$ is a member of $G$.

Proof. The proof depends upon the recognition of the fact that our situation is only slightly more general than that encountered by Mac Nerney in [8]. There is no problem if $u \leq v$. Suppose that $[v, u]$ is a subinterval of $S$ and each of $\left(g_{1}, m_{1}\right)$ and $\left(g_{2}, m_{2}\right)$ is in $\mathcal{G}_{\text {. If }} w$ is a positive number in $S$ and $w \geq u$ then $\pi_{w}\left(\nearrow(u, v)\left(g_{1}, m_{1}\right)-\mho(u, v)\left(g_{2}, m_{2}\right)\right) \leq\left\{k_{w}(u)-k_{w}(v)\right\} \pi_{w}\left(\left(g_{1}, m_{1}\right)-\left(g_{2}, m_{2}\right)\right)$.

From Lemma 1.2 of [8], we have that for each positive number $b$ there is an order partition $s$ of $\{u, v\}$ such that if $t$ refines $s$ then

$$
\left.\pi_{w}\left(\prod_{s}[1+\mho]\right](g, 1)-\prod_{t}[1+\mathcal{Q}](g, 1)\right)=N_{w}\left(\prod_{s}[1+V] g-\prod_{t}[1+V] g\right)<b .
$$

Hence the existence of $\Pi^{v}[1+V] g$ follows from the completeness of $G$.

Let $W$ denote the function from $S \times S$ into $H$ defined by $W(u, v) g=$ $\Pi^{v}[1+V] g$, for each $(u, v)$ in $S \times S$ and $g$ in $G$, and $b$ the function from $S$ into $X$ defined by

$$
b(u)= \begin{cases}f(u) & \text { if } u \leq 0 \\ {[W(u, 0) f](u)} & \text { if } 0 \leq u\end{cases}
$$

for each $u$ in $S^{\prime}$.

Theorem 2. $b$ is in $G$ and $[K(0, u) b](u)=\left[(R) \int_{u}^{0} V W[, 0] f\right](u)$, for each $u$ in $S^{\prime}$, i.e., $b$ is a solution of equation (1).

Proof. Let $\hat{g}$ denote the function from $S$ into $G$ defined by $\hat{g}(u)=W(u, 0) f$, for each $u$ in $S$. The net $\{S, \hat{g}\}$ is Cauchy and $\hat{g}_{u}(v)=b(v)$, for each $u$ in $S$ and $v$ in $S^{\prime}$ not exceeding $u$. Hence $b$ is in $G$.

Let $T$ be a positive number in $S$ and $\mathcal{W}$ denote the function from $S \times S$ into $\mathcal{H}$ defined by $\mathcal{C}(u, v)(g, m)={ }_{u} \Pi^{v}[1+\mathcal{C}](g, m)$, for each $(u, v)$ in $S \times S$ and $(g, m)$ in $\mathfrak{G}_{\times} \mathcal{G}$. We should note that $\mathcal{L}(u, v)(f, 1)=(W(u, v) f, 1)$, for each $(u, v)$ in $S \times S$. Hence, for $u, v$, and $w$ in $[0, T]$ with $u \leq v$, we have

$$
\begin{aligned}
N_{w}(W(v, 0) f-W(u, 0) f) & =\pi_{w}(\mathcal{W}(v, 0)(f, 1)-\mathcal{W}(u, 0)(f, 1)) \\
& =\Re_{w}(\mathcal{W}(v, u)(i)(u, 0)(f, 1)-\mathcal{W}(u, 0)(f, 1)) \\
& \leq(\mu(v, u)-1) \pi_{w}(\mathcal{W}(u, 0)(f, 1)) \\
& \leq(\mu(v, u)-1) \mu(u, 0)\left(N_{w}(f)+1\right) \\
& =(\mu(v, 0)-\mu(u, 0))\left(N_{w}(f)+1\right),
\end{aligned}
$$


where $\mu(x, y)={ }_{x} \Pi^{y}\left[1+d k_{T}\right]$ for each $(x, y)$ in $S \times S$.

Suppose that $u$ is in $[0, T]$ and $\epsilon$ is a positive number. There is an order partition $r$ of $\{u, 0\}$ such that if $\left\{s_{p}\right\}_{0}^{n}$ refines $r$ then

$$
N_{T}\left((R) \int_{u}^{0} V W[, 0] f-(R) \sum_{s} V W[, 0] f\right)<\frac{\epsilon}{3}
$$

and

$$
\text { (R) } \sum_{t} \mu[, 0] d k_{T}-(R) \sum_{s} \mu[, 0] d k_{T}<\frac{\epsilon}{3}\left(N_{T}(f)+1\right),
$$

for each refinement $t$ of $s$. Let $\bar{s}(p)=s(n-p)$, for $p=0,1,2, \ldots, n$, and $t$ be a refinement of $\bar{s}$ such that if $\alpha$ is an increasing integer-valued sequence and $\bar{s}=t[\alpha]$ then

$$
\begin{aligned}
& \sum_{p=1}^{n}(L) \int_{\bar{s}(p-1)}^{\bar{s}(p)} N_{x}\left(W\left(\bar{s}_{p-1}, 0\right) f-b\right) d k_{T}(x) \\
& -\sum_{p=1}^{n} \sum_{q=a(p-1)+1}^{a(p)} N_{t(q-1)}\left(W\left(\bar{s}_{p-1}, 0\right) f-b\right)\left(k_{T}\left(t_{q}\right)-k_{T}\left(t_{q-1}\right)\right)<\frac{\epsilon}{3} .
\end{aligned}
$$

Thus

$$
\begin{aligned}
N_{T}( & \left.(R) \int_{u}^{0} V W[, 0] f-K(0, u) b\right) \\
\leq & N_{T}\left((R) \int_{u}^{0} V W[, 0] f-(R) \sum_{s} V W[, 0] f\right) \\
& +N_{T}\left(\sum_{p=1}^{n} K\left(s_{p}, s_{p-1}\right) W\left(s_{p}, 0\right) f-K(0, u) b\right) \\
< & \frac{\epsilon}{3}+N_{T}\left(\sum_{p=1}^{n} K\left(\bar{s}_{p-1}, \bar{s}_{p}\right) W\left(\bar{s}_{p-1}, 0\right) f-K(0, u) b\right) \\
\leq & \frac{\epsilon}{3}+\sum_{p=1}^{n}(L) \int_{\bar{s}(p-1)}^{\bar{s}(p)} N_{x}\left(W\left(\bar{s}_{p-1}, 0\right) f-b\right) d k_{T}(x) \\
< & \frac{2 \epsilon}{3}+\sum_{p=1}^{n} \sum_{q=a(p-1)+1}^{\alpha(p)} N_{t(q-1)}\left(W\left(\bar{s}_{p-1}, 0\right) f\right. \\
\leq & \frac{2 \epsilon}{3}+\left\{(L) \sum_{t} \mu[, 0] d k_{T}-(L) \sum_{\bar{s}} \mu[, 0] d k_{T}\right\}\left(N_{T}(f)+1\right) \\
< & \quad \epsilon_{0}
\end{aligned}
$$


Therefore $N_{v}\left((R) \int_{u}^{0} V W[, 0] f-K(0, u) b\right)=0$ for each $u$ and $v$ in $S$ and so $(R) \int_{u}^{0} V W[, 0] f=K(0, u) b$, for each $u$ in $S$. Hence $\left[(R) \int_{u}^{0} V W[, 0] f\right](u)=$ $[K(0, u) b](u)$, for each $u$ in $S$.

Theorem 3. Equation (1) bas only one solution in G.

Proof. Suppose that each of $b_{1}$ and $b_{2}$ is a solution of equation (1) in $G$ and $u$ is a positive number in $S$. Then

$$
\begin{aligned}
N_{v}\left(b_{1}-b_{2}\right) & =N_{v}\left(K(0 ; u) b_{1}-K(0, u) b_{2}\right) \\
& \leq(L) \int_{0}^{v} N_{x}\left(b_{1}-b_{2}\right) d k_{u}(x) \leq N_{u}\left(b_{1}-b_{2}\right)\left\{k_{u}(v)-k_{u}(0)\right\},
\end{aligned}
$$

for each $v$ in $S$ not exceeding $u$. Therefore, for each positive integer $n$,

$$
N_{u}\left(b_{1}-b_{2}\right) \leq N_{u}\left(b_{1}-b_{2}\right)\left[k_{u}(u)-k_{u}(0)\right]^{n} / n !
$$

and so $N_{u}\left(b_{1}-b_{2}\right)=0$. This means $b_{1}=b_{2}$.

Continuity. For each positive number $t$ in $S$ let $\mu_{t}$ denote the function from $S \times S$ to the nonnegative numbers defined by $\mu_{t}(u, v)=\prod_{u} \Pi^{v}\left[1+\left|d k_{t}\right|\right]$. Then we have for each positive number $t$ in $S$, subinterval $[u, v]$ of $S$, and $\left(g_{1}, g_{2}\right)$ in $G \times G$, that $N_{t}\left(W(u, v) g_{1}-W(u, v) g_{2}\right) \leq \mu_{t}(u, v) N_{t}\left(g_{1}-g_{2}\right)$. This observation serves as a proof for the following theorem.

Theorem 4. If $\{D, \hat{f}\}$ is a Cauchy net in $G$ with limit $f$ and $\hat{b}_{n}$, for each $n$ in $D$, is the member of $G$ such that $\hat{b}_{n}(u)=\hat{f}_{n}(u)+\left[K(0, u) \hat{b}_{n}\right](u)$, for each $u$ in $S$, then $\{D, \hat{b}\}$ bas limit $b$ in $G$.

Theorem 5. If $S=[0, T] ; D$ is a directed set; $\hat{K}$ is a function from $D$ into the class of all functions from $S \times S$ into $H$; and $\hat{k}$ is a function from $D$ into the set of nondecreasing functions on $S$ such that (1) $\hat{K}_{n}$ satisfies (i) and (ii) above, for each $n$ in $D$, (2) $\hat{k}_{n}$ is a super function for $\hat{K}_{n}$ for each $n$ in $D$, $\left(g_{1}, g_{2}\right)$ in $G \times G$, and subinterval $[u, v]$ of $S$, (3) $k_{T}$ is the uniform limit of $\{D, \hat{k}\}$; and (4) for each positive number $b$ and $g$ in $G$ there is a member $n$ of $D$ such that $N_{T}\left(K(u, v) g-\hat{K}_{m}(u, v) g\right)<b$, for each $m$ in $D$ which follows $n$ and subinterval $[u, v]$ of $S$; then $\{D, \hat{b}\}$, where $\hat{b}_{n}$ for each $n$ in $D$ is the member of G such that $\hat{b}_{n}(u)=f(u)+\left[\hat{K}_{n}(0, u) \hat{b}_{n}\right](u)$, for each $u$ in $S$, bas limit $b$ in $G$.

Proof. Theorem 5 is a corollary of Theorem 3 [12]. For each $n$ in $D$, let $\hat{F}_{n}$ denote the function from $S \times S$ into $H$ defined by $\left[\hat{F}_{n}(u, v) g\right](w)=$ $\left[\hat{K}_{n}(0, v) g\right](w)$. Let $F$ denote the function from $S \times S$ into $H$ defined by $[F(u, v) g](w)=[K(0, v) g](w)$. Then $\{D, \hat{F}\}$ is convergent $w$ ith limit $F$. Hence $\{D, \hat{b}\}$ converges uniformly on $S$ with limit $h$. 
Realizations. The realizations of $K$ presented in this section will demonstrate the wide variety of hereditary systems to which the methods of product integration apply. Suppose that $S=S^{\prime}, G$ is the class of quasicontinuous functions from $S$ into $X$, and $N_{u}(f)$, for each $u$ in $S$ and $f$ in $G$, is the least number $b$ such that $\|f(x)\| \leq b$ for all $x$ in $S$ not exceeding $u$. Let $M$ denote the class of all functions from $X$ into $X$. Suppose that $F$ is a function from $S \times S$ into $M$ and $k$ is a nondecreasing function from $S$ to the nonnegative numbers such that (i) $F[, u] P$ is in $G$, for each $u$ in $S$ and $P$ in $X$, (ii) $\|F(u, v) 0-F(u, w) 0\| \leq|k(v)-k(w)|$ and

$$
\|\{F(u, v)-F(u, w)\} P-\{F(u, v)-F(u, w)\} Q\| \leq|k(u)-k(v)| \cdot\|P-Q\|,
$$

for all $u, v$, and $w$ in $S$ and $(P, Q)$ in $X \times X$. The equation

$$
b(u)=f(u)+(L) \int_{0}^{u} d F[u,] h,
$$

where $f$ is in $G$, was considered in [11]. We can relate those results to the present paper by introducing a function $K$ from $S \times S$ into $H$ as follows:

$$
[K(u, v) g](t)= \begin{cases}(L) \int_{u}^{v} d F[t,] g & \text { if } u \leq v \leq t \\ (L) \int_{u}^{t} d F[t,] g & \text { if } u \leq t \leq v \\ 0 & \text { otherwise. }\end{cases}
$$

With this realization, equations (1) and (2) are equivalent.

Mac Nerney [7] and Herod [5] have both introduced generalizations of Stieltjes integral equations in the direction of hereditary systems. Comparison of our paper with each of theirs might illuminate condition (iii) on $K$.

For our second realization, suppose that $X=R^{n},\|\cdot\|$ is the Euclidean norm, $S^{\prime}=S, G$ is the class of quasicontinuous functions from $S$ into $X$, and $N_{u}(f)$, for each $u$ in $S$ and $f$ in $G$, is the least number $b$ such that $\|f(x)\| \leq b$ for all $x$ in $S$ not exceeding $u$. Suppose that $g$ is a function from $S \times S \times X$ into $X$ and $m$ is a function from $S \times S$ into the nonnegative numbers such that

(i) for each $u$ in $S$ and $f$ in $G$, the function $b$ from $S$ into $X$ defined by $b(v)=g(u, v, f(v))$ is Lebesgue summable on each compact subinterval of $S$,

(ii) for each subinterval $[u, v]$ of $S$ and $f$ in $G$ the function $\int_{u}^{v} g[, s, f(s)] d s$ is in $G$,

(iii) $m[u$,$] is summable on each compact subinterval of S$, for each $u$ in $S$,

(iv) $\|g(u, s, 0)\| \leq m(u, s)$ and $\|g(u, s, P)-g(u, s, Q)\| \leq m(u, s)\|P-Q\|$, for each $u$ in $S$, almost all $s$ in $S$, and $(P, Q)$ in $X \times X$, and 
(v) there is a nondecreasing function $k$ from $S$ to the nonnegative numbers such that $\int_{u}^{v} m(w, s) d s \leq k(v)-k(u)$, for each subinterval $[n, v]$ of $S$ and $w$ in $S$. The equation

$$
b(u)=f(u)+\int_{0}^{u} g(u, s, b(s)) d s,
$$

where $f$ is in $G$, is seen to be equivalent to equation (1) upon defining $K$ as follows:

$$
[K(u, v) b](t)= \begin{cases}\int_{u}^{v} g(t, s, b(s)) d s & \text { if } u \leq v \leq t \\ \int_{u}^{t} g(t, s, b(s)) d s & \text { if } u \leq t \leq v \\ 0 & \text { otherwise. }\end{cases}
$$

Finally, suppose that $\{X,\|\cdot\|\}$ is either the space of real numbers or the space of complex numbers with the usual norm, $r$ is a positive number, $S$ is a compact interval $[0, T]$, and $S^{\prime}=[-r, T]$. Let $G$ be the class of all absolutely continuous functions from $S^{\prime}$ into $X$ and let $N_{u}(f)=1$.u.b. $-r \leq x \leq u\|f(x)\|+$ $\int_{-r}^{u}\|d f\|$. Suppose that $m$ is a summable function with values in the nonnegative numbers, $g_{1}$ is a summable function with values in $X$, and $g_{2}$ is a function from $S \times[-r, 0]$ into $X$ such that

(i) $g_{2}[u, I]$ is Lipschitz with Lipschitz constant $m(u)$, for each $u$ in $S$, and

(ii) for each $f$ in $G$, the function $b$ from $S$ into $X$ defined by $b(u)=$ $\int_{-r}^{0} d f[u+I] d g_{2}[u, I] / d I$ is summable.

The reader is referred to [2] for a discussion of the representation of continuous linear functionals on the space of absolutely continuous functions.

The equation

$$
b(u)=f(u)+\int_{0}^{u} g_{1}(s) b(s-r) d s+\int_{0}^{u}\left\{\int_{\sim r}^{0} \frac{d b[s+I] d g_{2}[s, I]}{d I}\right\} d s,
$$

where $f$ is in $G$, is seen to be equivalent to equation (1) upon defining $K$ as follows:

$[K(u, v) b](t)= \begin{cases}\int_{u}^{v} g_{1}(s) b(s-r) d s+\int_{u}^{v}\left\{\int_{-r}^{0} \frac{d b[s+I] d g_{2}[s, I]}{d I}\right\} d s \quad \text { if } u \leq v \leq t, \\ \int_{u}^{t} g_{1}(s) b(s-r) d s+\int_{u}^{v}\left\{\int_{-r}^{0} \frac{d b[s+I] d g_{2}[s, I]}{d I}\right\} d s & \text { if } u \leq t \leq v, \\ 0 & \text { otherwise. }\end{cases}$ 
Local theory. Condition (iii) on $K$ is the critical assumption which permits the application of Mac Nerney's product integral theory to hereditary systems. Other researchers have studied the product integral [1], [10], [6] and this raises the possibility of weakening or changing condition (iii). A complete investigation of this problem is outside the scope of this paper; but in this section, two alternate versions of condition (iii) are presented which fit within the framework of the existence theory already developed.

We will assume in this section that, for each $u$ in $S$ and $f$ in $G, N_{u}(f)$ is the least number $b$ such that $\|f(x)\| \leq b$ for all $x$ in $S^{\prime}$ not exceeding $u$. For each positive number $b$ let $T_{b}$ denote the function from $X$ into $X$ defined by

$$
T_{b} P= \begin{cases}P & \text { if }\|P\| \leq b, \\ (b /\|P\|) P & \text { if }\|P\| \geq b,\end{cases}
$$

for each $P$ in $X$. We will assume, further, that $T_{b}[f]$ is a member of $G$, for each positive number $b$ and $f$ in $G$. Note that $N_{u}\left(T_{b}[f]-T_{b}[g]\right) \leq 2 N_{u}(f-g)$, for each $u$ in $S$, positive number $b$, and $(f, g)$ in $G \times G$.

Suppose that $K_{1}$ is a function from $S^{\prime} \times S^{\prime}$ into $H$ which satisfies conditions (i) and (ii) of the first section and

(iv) for each positive number $b$ the function $K$ from $S \times S$ into $H$ defined by $K(u, v) g=K_{1}(u, v) T_{b}[g]$ has a family of continuous super functions.

Let $V_{1}$ denote the function from $S \times S$ into $H$ defined as follows:

$$
\left[V_{1}(u, v) f\right](w)= \begin{cases}{\left[K_{1}(v, u) f\right](w)} & \text { if } v \leq u, \\ 0 & \text { otherwise }\end{cases}
$$

for each $f$ in $G,(u, v)$ in $S \times S$, and $w$ in $S^{\prime}$.

Theorem 6. For each $f$ in $G$ there is a positive number $v$ in $S$ such that, for each $u$ in $S$ not exceeding $v, W_{1}(u, 0) f={ }_{u} \Pi^{0}\left[1+V_{1}\right]$ f exists. Furthermore, if $b$ is the function from $S^{\prime}$ into $X$ defined by $b(u)=\left[W_{1}(v, 0) f\right](u)$, for all $u$ in $S^{\prime}$, then $b$ is in $G$ and $b(u)=f(u)+\left[K_{1}(u, 0) b\right](u)$, for all $u$ in $S^{\prime}$ not exceeding $v$.

Proof. Suppose that $w$ is a positive number in $S, b$ is a number larger than $N_{w}(f)+1, k$ is a function from $S$ into the class of continuous nondecreasing functions from $S$ to the numbers corresponding to be as in condition (iv), and $v$ is a positive number in $[0, w]$ such that $\exp \left(2 k_{w}(v)-2 k_{w}(u)\right) \leq$ $b /\left\{N_{w}(f)+1\right\}$. Let $V_{b}$ be the function from $S \times S$ into $H$ defined by $V_{b}(u, v) f$ $=V_{1}(u, v) T_{b}[f]$, for each $(u, v)$ in $S \times S$ and $g$ in $G$. Define $\vartheta_{1}$ and $\mho_{b}$ in terms of $V_{1}$ and $V_{b}$, respectively, as in $\$ 2$. For each decreasing sequence $\left\{t_{p}\right\}_{0}^{n}$ such that $t_{0} \leq v$ and $t_{n}=0$, we have 


$$
\begin{aligned}
\pi_{w}\left(\prod_{t}\left[1+\mathcal{O}_{b}\right](f, 1)\right) & \leq \prod_{t}\left(1+2 d k_{w}\right) \Re_{w}((f, 1)) \\
& \leq \exp \left(2 k_{w}(v)-2 k_{w}(u)\right)\left(N_{w}(f)+1\right) \leq b .
\end{aligned}
$$

Hence $N_{w}\left(\Pi_{t}\left[1+V_{b}\right] f\right) \leq b$. So, by induction, $\Pi_{t}\left[1+V_{b}\right] f=\Pi_{t}\left[1+V_{1}\right] f$ and hence $\Pi^{0}\left[1+V_{1}\right] f$ exists for each $u$ in $S$ not exceeding $v$. This also shows that $W_{1}(v, 0) f=W_{b}(v, 0) f$. If $b=W_{1}(v, 0) f$, then $b$ is in $G, N_{w}(b) \leq b$, and $b(u)=f(u)+\left[K_{b}(u, 0) b\right](u)=f(u)+\left[K_{1}(u, 0) b\right](u)$, for all $u$ in $S^{\prime}$ not exceeding $v$.

For the remainder of the section, we will assume that $G$ contains the constant functions and each member of $G$ is continuous on $S$. Suppose that $P$ is a point in $X$ and $K_{2}$ is a function from $S^{\prime} \times S^{\prime}$ into $H$ which satisfies conditions (i) and (ii) of the first section and

(v) there is a positive number $b$ such that the function $K$ from $S^{\prime} \times S^{\prime}$ into $H$ defined by $K(u, v) g=K_{2}(u, v)\left\{T_{b}\left[g-1_{s^{\prime}} P\right]+1_{s^{\prime}} P\right\}$ has a family of continuous super functions.

Let $V_{2}$ denote the function from $S \times S$ into $H$ defined as follows:

$$
\left[V_{2}(u, v) f\right](w)= \begin{cases}{\left[K_{2}(v, u) f\right](w)} & \text { if } v \leq u, \\ 0 & \text { otherwise }\end{cases}
$$

for each $f$ in $G,(u, v)$ in $S \times S$, and $w$ in $S^{\prime}$.

Theorem 7. There is a positive number $b$ such that if $f$ is in $G$ and $N_{0}\left(f-1_{S^{\prime}} P\right)<b$ then for some positive number $v$ in $S$ the product $W_{2}(u, 0) f=$ ${ }_{u} \Pi^{0}\left[1+V_{2}\right] f$ exists, for each $u$ in $S$ not exceeding $v$. Furthermore, if $b$ is the function from $S^{\prime}$ into $X$ defined by $b(u)=\left[W_{2}(v, 0) f\right](u)$, for all $u$ in $S^{\prime}$, then $b$ is in $G$ and $b(u)=f(u)+\left[K_{2}(u, 0) b\right](u)$, for all $u$ in $S^{\prime}$ not exceeding $v$.

A proof may be constructed along the lines of the proof of the preceding theorem by introducing $V_{b}$ as follows: $V_{b}(u, v) g=V_{2}(u, v)\left\{T_{b}\left[g-1_{s^{\prime}} P\right]+1_{S^{\prime}} P\right\}$, for $(u, v)$ in $S \times S$ and $g$ in $G$. The inequality [8]

$$
\begin{aligned}
N_{v}\left(\prod_{t}[1\right. & \left.\left.+v_{b}\right] f-1_{s^{\prime}} P\right) \\
& \leq \pi_{v}\left(\sum_{p} \mho_{b}\left(t_{p-1}, t_{p}\right) \prod_{q=p+1}^{n}\left[1+\bigcup_{b}\left(t_{q-1}, t_{q}\right)\right](f, 1)\right)+N_{v}\left(f-1 s^{\prime} P\right) \\
& \leq\left\{\exp \left(2 k_{w}(v)-2 k(0)\right)-1\right\}\left(N_{v}(f)+1\right)+N_{v}\left(f-1_{s^{\prime}} P\right)
\end{aligned}
$$

leads us to choose $v$ so that 


$$
\left\{\exp \left(2 k_{w}(v)-2 k_{w}(0)\right)-1\right\}\left(N_{v}(f)+1\right)+N_{v}\left(f-1 s^{\prime} P\right) \leq b .
$$

Corollary. If $K_{2}(u, v) 0=0$, for each $(u, v)$ in $S^{\prime} \times S^{\prime}, P=0$, and $k$ has only one value and that value has a bounded final set, then there is a positive number $d$ such that if $f$ is in $G$ and $N_{w}(f) \leq d$, for each $w$ in $S$, then $W_{2}(u, 0) f$ $={ }_{u} \Pi^{0}\left[1+V_{2}\right]$ f exists for each $n$ in $S$. Furthermore, if $b$ is the function from $S^{\prime}$ into $X$ defined by

$$
b(u)= \begin{cases}f(u) & \text { if } u \leq 0, \\ {\left[W_{2}(u, 0) f\right](u)} & \text { if } 0 \leq u,\end{cases}
$$

for each $u$ in $S^{\prime}$, then $b$ is in $G$ and $b(u)=f(u)+\left[K_{2}(u, 0) b\right](u)$, for all $u$ in $S^{\prime}$.

More on realizations. Suppose that $X=R^{n},\|\cdot\|$ is the Euclidean norm, $r$ is a positive number, $S$ is a compact interval $[0, T], S^{\prime}=[-r, T], G$ is the class of continuous functions from $S^{\prime}$ into $X$, and $N_{u}(f)$, for each $u$ in $S$ and $f$ in $G$, is the least number $b$ such that $\|f(x)\| \leq b$, for all $x$ in $S^{\prime}$ not exceeding $u$. Let $C$ denote the continuous functions from $[-r, 0]$ into $X$ and $\|\cdot\|$, the supremum norm on $C$. For each $u$ in $S$ and $f$ in $G$, let $f_{u}$ denote the member of $C$ defined by $f_{u}(x)=f(u+x)$, for all $x$ in $[-r, 0]$.

Suppose that $X_{0}$ is an open subset of $X, C_{0}$ denotes the members of $C$ with values in $X_{0}$, and $g$ is a function from $X \times X \times C_{0}$ into $X$ with the following properties.

(i) For each $f$ in $G$ with values in $X_{0}$ and $u$ in $S$ the function $b$ from $S$ into $X$ defined by $b(v)=g\left(u, v, f_{v}\right)$ is summable.

(ii) For each subinterval $[u, v]$ of $S$ and $f$ in $G$ with values in $X_{0}$ the function $\int_{u}^{v} g\left[, s, f_{s}\right] d s$ is in $G$.

(iii) For each closed ball $B$ contained in $X_{0}$ there is a function $m$ from $S \times S$ to the nonnegative numbers and a continuous nondecreasing function $k$ from $S$ such that $\|g(u, s, f)\| \leq m(u, s)$ and $\|g(u, s, f)-g(u, s, g)\| \leq$ $m(u, s)\|f-g\|_{1}$, for each $u$ in $S$, almost all $s$ in $S$, and $f$ and $g$ in $C_{0}$ with values in $B ; m[u$,$] is summable, for each u$ in $S$; and $\int_{u}^{v} m(w, s) d s \leq$ $k(v)-k(u)$, for each subinterval $[u, v]$ of $S$ and $w$ in $S$.

We want to relate the functional integral equation

$$
b(u)= \begin{cases}f(u) & \text { if }-r \leq u \leq 0, \\ f(u)+\int_{0}^{u} g\left(u, s, b_{s}\right) d s & \text { if } 0 \leq u,\end{cases}
$$

where $f$ is in $C_{0}$, to the development in the preceding section. Suppose that $P$ is a point in $X_{0}$ and $b$ is a positive number such that the closed ball $B$ with 
center $P$ and radius $r$ lies in $X_{0}$. Suppose that $m$ and $k$ are functions as in (iii), above. Let $K_{2}$ denote the function from $S^{\prime} \times S^{\prime}$ into $H$ defined as follows:

$$
\left[K_{2}(u, v) b\right](w)=\left\{\begin{array}{l}
\int_{x}^{y} g\left(w, s, T_{b}\left[b_{s}-1_{[-r, 0}\right]^{P]}+1_{[-r, 0]}^{P) d s} \text { if } u \leq v,\right. \\
0 \text { otherwise, }
\end{array}\right.
$$

where $y=\max (0, \min (v, w))$ and $x=\max (0, \min (u, w))$.

If $f$ in equation (5) satisfies $N_{0}\left(f-1_{[-\tau, T} P\right) \leq b$ then (5) is equivalent to $b(u)=f(u)+\left[K_{2}(0, u) b\right](u)$ and by Theorem 7 has a solution on $[-r, v]$ for some $v$ in $[0, T]$ which can be represented as a product integral.

\section{BIBLIOGRAPHY}

1. J. R. Dorroh, Some classes of semigroups of nonlinear transformations and the ir generators, J. Math. Soc. Japan 20 (1968), 437-455. MR 37 \#6796.

2. J. R. Edwards and S. G. Wayment, Representations for transformations continuous in the BV norm, Trans. Amer. Math. Soc. 154 (1971), 251-265. MR 43 \#466.

3. J.K. Hale, Functional differential equations, Springer-Verlag, New York, 1971.

4. J. K. Hale and M. A. Cruz, Existence, uniqueness and continuous dependence for hereditary systems, Ann. Mat. Pura Appl. (4) 85 (1970), 63-81. MR 41 \#7238.

5. J. V. Herod, Solving integral equations by iteration, Duke Math. J. 34 (1967), 519-534. MR $36 \# 625$.

6. - A pairing of a class of evolution systems with a class of generators, Trans. Amer. Math. Soc. 157 (1971), 247-260. MR 43 \#6778.

7. J. S. Mac Nerney, A linear initial-value problem, Bull. Amer. Math. Soc. 69 (1963), 314-329. MR $26 \# 4133$.

8. - A nonlinear integral operation, Illinois J. Math. 8 (1964), 621-638. MR $29 \# 5082$.

9. E. J. McShane, Stochastic integrals and stochastic functional equations, SIAM J. Appl. Math. 17 (1969), 287-306. MR 39 \#7691.

10。 J.W. Neuberger, Product integral formulae for nonlinear expansive semigroups and non-expansive evolution systems, J. Math. and Mech. 19 (1969/70), 403-409. MR $40 \$ 6301$.

11. J. A. Reneke, A product integral solution of a Stieltjes-Volterra integral equation, Proc. Amer. Math. Soc. 24 (1970), 621-626. MR 40 \#6214.

12. - Continuity for Stieltjes-Volterra integral equations, Rev. Roumaine Math. Pures Appl. 17 (1972), 389-401.

DEPARTMENT OF MATHEMATICS, UNIVERSITY OF HOUSTON, HOUSTON TEXAS 77004

Current address: Department of Mathematical Sciences, Clemson University, Clemson, South Carolina 29631 